\title{
Analysis, Adaptive Control and Synchronization of a Seven-Term Novel 3-D Chaotic System with Three Quadratic Nonlinearities and its Digital Implementation in LabVIEW
}

\author{
S. Vaidyanathan*,1, K. Rajagopal ${ }^{2}$, Ch. K. Volos ${ }^{3}$, I. M. Kyprianidis ${ }^{3}$ and I. N. Stouboulos ${ }^{3}$ \\ ${ }^{I}$ Research and Development Centre, Vel Tech University, Avadi, Chennai-600062, Tamil Nadu, India. \\ ${ }^{2}$ Department of Electronics Engineering, Defense Engineering College, Ethiopia \\ ${ }^{3}$ Department of Physics, Aristotle University of Thessaloniki, Thessaloniki, GR-54124, Greece.
}

Received 8 September 2014; Revised 13 October 2014; Accepted 25 October 2014

\begin{abstract}
This research work proposes a seven-term novel 3-D chaotic system with three quadratic nonlinearities and analyses the fundamental properties of the system such as dissipativity, symmetry, equilibria, Lyapunov exponents and Kaplan-Yorke dimension. The phase portraits of the novel chaotic system simulated using MATLAB depict the strange chaotic attractor of the novel system. For the parameter values and initial conditions chosen in this work, the Lyapunov exponents of the novel chaotic system are obtained as $L_{1}=2.71916, L_{2}=0$ and $L_{3}=-13.72776$. Also, the KaplanYorke dimension of the novel chaotic system is obtained as $D_{K Y}=2.19808$. Next, an adaptive controller is designed to stabilize the novel chaotic system with unknown system parameters. Also, an adaptive controller is designed to achieve global chaos synchronization of two identical novel chaotic systems with unknown system parameters. Finally, an electronic circuit realization of the novel chaotic system is depicted using LabVIEW to confirm the feasibility of the theoretical chaotic model.
\end{abstract}

Keywords: Chaos, chaotic systems, synchronization, Lyapunov exponents, Kaplan-Yorke dimension, circuit simulation.

\section{Introduction}

Chaotic systems are commonly defined as nonlinear dynamical systems which are very sensitive to initial conditions, topologically mixing and also with dense periodic orbits [1]. There is great interest in the chaos literature in the discovery of chaotic behavior in nature and physical systems.

Historically, Poincare was the first to notice the possibility of chaos when he was working on a three-body problem. A significant development also occurred when Lorenz discovered a 3-D chaotic system of a weather model [2]. Since then, many paradigms of 3-D chaotic systems have been found in the chaos literature such as Rössler system [3], Rabinovich system [4], ACT system [5], Sprott systems [6], Chen system [7], Lü system [8], Shaw system [9], Feeny system [10], Shimizu system [11], Liu-Chen system [12], Cai system [13], Tigan system [14], Colpitt's oscillator [15], WINDMI system [16], Zhou system [17], etc.

Recently, many 3-D chaotic systems have been discovered such as Li system [18], Elhadj system [19], Pan system [20], Sundarapandian system [21], Yu-Wang system [22], Sundarapandian-Pehlivan system [23], Zhu system [24], Vaidyanathan systems [25-31], VaidyanathanMadhavan system [32], Pehlivan-Moroz-Vaidyanathan system [33], Jafari system [34], Pham system [35], etc.

The study of chaos theory has many important applications in science and engineering such as oscillators [36-37], lasers [38,39], robotics [40-43], chemical reactors $[44,45]$, biology $[46,47]$, ecology $[48,49]$, cardiology [50], memristors [51-53], neural networks [54-56], secure communications [57-60], cryptosystems [61-64], economics [65-67], etc.

Chaos control and chaos synchronization are important research problems in the chaos theory. In the last three decades, many mathematical methods have been developed successfully to address these research problems.

The study of control of a chaotic system investigates methods for designing feedback control laws that globally or locally asymptotically stabilize or regulate the outputs of a chaotic system.

\footnotetext{
* E-mail address: sundarvtu@gmail.com ISSN: 1791-2377 @ 2015Kavala Institute of Technology. All rights reserved.
} 
Many methods have been developed for the control and tracking of chaotic systems such as active control [68-72], adaptive control [73-79], backstepping control [80-82], sliding mode control [83, 84], etc.

Chaos synchronization problem deals with the synchronization of a couple of systems called the master or drive system and the slave or response system. To solve this problem, control laws are designed so that the output of the slave system tracks the output of the master system asymptotically with time. Because of the butterfly effect, this is a challenging problem even when the initial conditions of the master and slave systems are nearly identical because of the exponential divergence of the outputs of the two systems in the absence of any control. The synchronization of chaotic systems has applications in secure communications [85-87], cryptosystems [88, 89], encryption [90, 91], etc.

In the chaos literature, many different methodologies have been also proposed for the synchronization and antisynchronization of chaotic systems such as PC method [92], active control [93-103], time-delayed feedback control [104,105], adaptive control [106-119], sampled-data feedback control [120-121], backstepping control [122-128], sliding mode control [129-134], etc.

In this research paper, we propose a seven-term novel chaotic system with three quadratic nonlinearities. The paper gives a detailed analysis of the fundamental properties of the chaotic system such as dissipativity, symmetry, equilibria, Lyapunov exponents, etc. The Lyapunov exponents of the novel chaotic system are found as $L_{1}=2.71916, L_{2}=0$ and $L_{3}=-13.72226$. The Kaplan-Yorke dimension of the novel chaotic system is found as $D_{\mathrm{KY}}=2.19808$.

Next, we derive an adaptive control law that stabilizes the 3-D novel chaotic system when the system parameters are unknown. The main control result is proved using Lyapunov stability theory and MATLAB simulations are shown to illustrate the stabilization of the chaotic system.

Furthermore, we derive an adaptive control law that asymptotically synchronizes the identical 3-D novel chaotic systems when the system parameters are unknown. The main synchronization result is proved using Lyapunov stability theory and MATLAB simulations are shown to illustrate the chaos synchronization of the identical novel chaotic systems.

Finally, an electronic circuit realization of the 3-D novel chaotic system using LabVIEW is presented to confirm the feasibility of the theoretical chaos model and control design.

\section{A Seven-Term 3-D Novel Chaotic System}

The dynamics of the seven-term novel 3-D chaotic system with three quadratic nonlinearities is described by

$$
\left\{\begin{array}{l}
\frac{d x_{1}}{d t}=a\left(x_{2}-x_{1}\right)+c x_{2} x_{3} \\
\frac{d x_{2}}{d t}=b x_{1}-x_{1} x_{3} \\
\frac{d x_{3}}{d t}=x_{1} x_{2}-x_{3}
\end{array}\right.
$$

where $x_{1}, x_{2}, x_{3}$ are the states and $a, b, c$ are positive parameters.

The nonlinear system (1) depicts a strange chaotic attractor when the parameter values are taken as:

$a=10, b=15, c=12$

We take the initial conditions as: $x_{1}(0)=0.6, x_{2}(0)=1.8, x_{3}(0)=1.2$

The 3-D portrait of the strange chaotic attractor (1) for the parameter values (2) and the initial conditions (3) is depicted in Fig. 1, and the 2-D portraits (projections on the three coordinate planes) are depicted in Figs. 2-4.

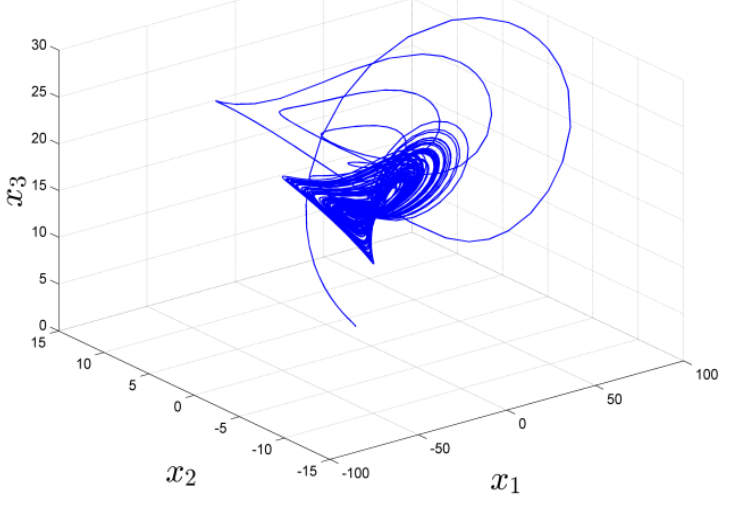

Fig. 1.The strange chaotic attractor of the novel chaotic system in $R^{3}$.

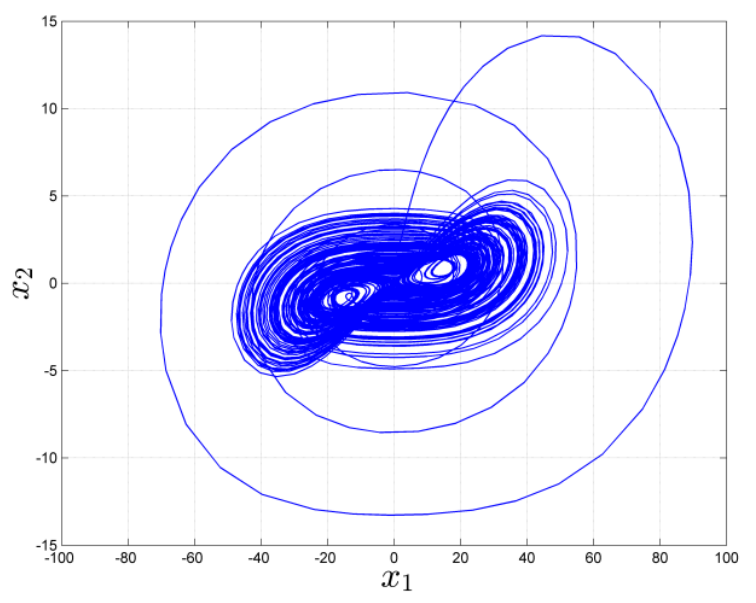

Fig. 2.The 2-D projection of the attractor on the $\left(x_{1}, x_{2}\right)$ plane.

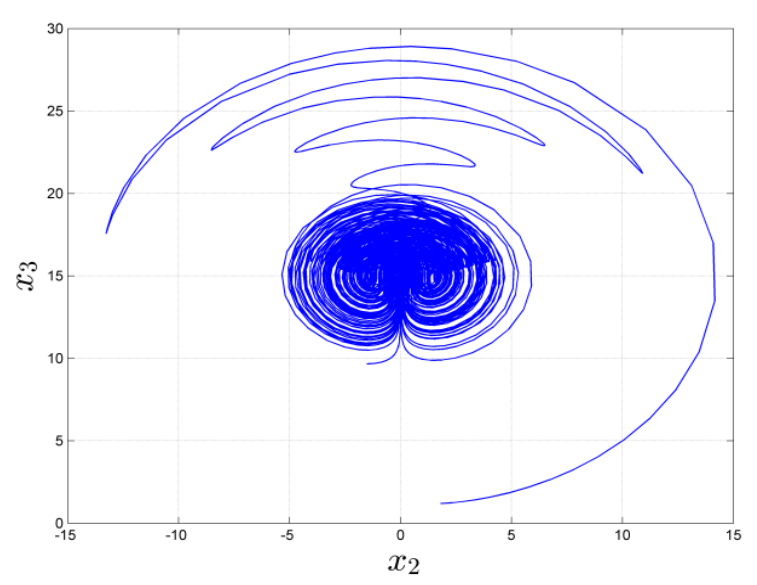

Fig. 3.The 2-D projection of the attractor on the $\left(x_{2}, x_{3}\right)$ plane. 


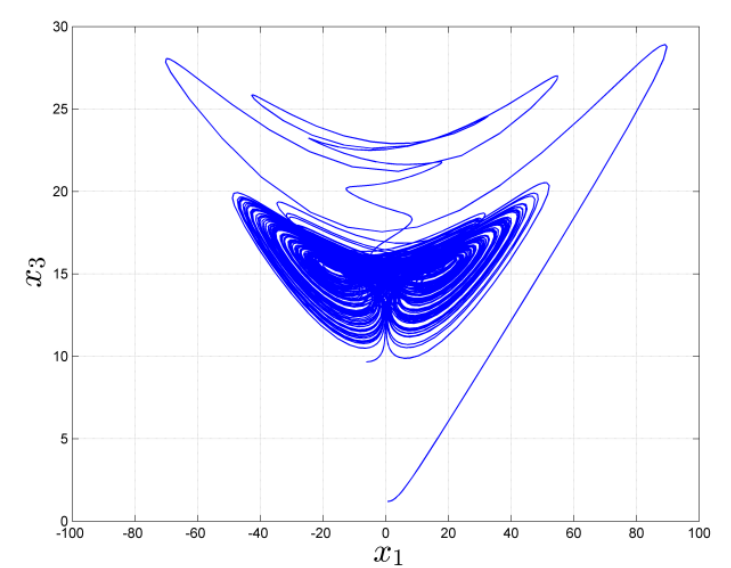

Fig. 4.The 2-D projection of the attractor on the $\left(x_{1}, x_{3}\right)$ plane.

\section{Analysis of the 3-D Novel Chaotic System}

In this section, qualitative properties of the 3-D novel chaotic system are detailed.

\subsection{Dissipativity}

In vector notation, we may express the system (1) as:

$\frac{d x}{d t}=f(\boldsymbol{x})=\left[\begin{array}{l}f_{1}\left(x_{1}, x_{2}, x_{3}\right) \\ f_{2}\left(x_{1}, x_{2}, x_{3}\right) \\ f_{3}\left(x_{1}, x_{2}, x_{3}\right)\end{array}\right]$

where

$\left\{\begin{array}{l}f_{1}\left(x_{1}, x_{2}, x_{3}\right)=a\left(x_{2}-x_{1}\right)+c x_{2} x_{3} \\ f_{2}\left(x_{1}, x_{2}, x_{3}\right)=b x_{1}-x_{1} x_{3} \\ f_{3}\left(x_{1}, x_{2}, x_{3}\right)=x_{1} x_{2}-x_{3}\end{array}\right.$

We take the parameter values as in the chaotic case, viz. $a=10, b=15$ and $c=12$.

Let $\Omega$ be any region in $\boldsymbol{R}^{3}$ with a smooth boundary and also $\Omega(t)=\Phi_{t}(\Omega)$, where $\Phi_{t}$ is the flow of $f$.

Furthermore, let $V(t)$ denote the volume of $\Omega(t)$.

By Liouville's theorem, we have

$$
\frac{d V}{d t}=\int_{\Omega(t)}(\nabla \cdot f) d x_{1} d x_{2} d x_{3}
$$

The divergence of the novel chaotic system (1) is easily found as:

$\nabla \cdot f=\frac{\partial f_{1}}{\partial x_{1}}+\frac{\partial f_{2}}{\partial x_{2}}+\frac{\partial f_{3}}{\partial x_{3}}=-a-1=-11$

Substituting (7) into (6), we obtain the first order ODE.

$$
\frac{d V}{d t}=-11 V(t)
$$

Integrating (8), we obtain the unique solution as:

$V(t)=\exp (-11 t) V(0)$
It is evident from Eq.(9) that $V(t) \rightarrow 0$ exponentially as $t \rightarrow \infty$.

This shows that the novel chaotic system (1) is dissipative.

Hence, the system limit sets are ultimately confined into a specific limit set of zero volume, and the asymptotic motion of the novel chaotic system (1) settles onto a strange attractor of the system.

\subsection{Symmetry and Invariance}

It is easy to see that the system (1) is invariant under the coordinates transformation

$\left(x_{1}, x_{2}, x_{3}\right) \rightarrow\left(-x_{1},-x_{2}, x_{3}\right)$

Thus, the system (1) has rotation symmetry about the $x_{3}$-axis and any non-trivial trajectory of the system (1) must have a twin trajectory. It is also easy to see that the $x_{3}$-axis is invariant under the flow of the system (1).

\subsection{Equilibrium Points}

The equilibrium points of the novel chaotic system (1) are obtained by solving the following system of equations (with $a=10, b=15$ and $c=12$ ).

$$
\left\{\begin{aligned}
a\left(x_{2}-x_{1}\right)+c x_{2} x_{3} & =0 \\
b x_{1}-x_{1} x_{3} & =0 \\
x_{1} x_{2}-c x_{3} & =0
\end{aligned}\right.
$$

The equilibria of (11) are calculated as

$E_{0}=\left[\begin{array}{l}0 \\ 0 \\ 0\end{array}\right], E_{1}=\left[\begin{array}{c}16.88194 \\ 0.88852 \\ 15.00000\end{array}\right], E_{2}=\left[\begin{array}{c}-16.88194 \\ -0.88852 \\ 15.00000\end{array}\right]$

The Jacobian matrix of the system (1) at $\boldsymbol{x}$ is given by

$$
J(\boldsymbol{x})=\left[\begin{array}{ccc}
-a & a+c x_{3} & c x_{2} \\
b-x_{3} & 0 & -x_{1} \\
x_{2} & x_{1} & -1
\end{array}\right]
$$

The Jacobian matrix at $E_{0}$ is obtained as:

$$
J_{0}=J\left(E_{0}\right)=\left[\begin{array}{ccc}
-10 & 10 & 0 \\
15 & 0 & 0 \\
0 & 0 & -1
\end{array}\right]
$$

Using MATLAB, we find the eigenvalues of $J_{0}$ as:

$$
\lambda_{1}=-18.2288, \lambda_{2}=-1, \lambda_{3}=8.2288
$$

Thus, the equilibrium $E_{0}$ is a saddle-point, which is unstable.

The Jacobian matrix at $E_{1}$ is obtained as:

$J_{1}=J\left(E_{1}\right)=\left[\begin{array}{ccl}-10 & 190 & 10.6622 \\ 0 & 0 & -16.8819 \\ 0.8885 & 16.8819 & -1\end{array}\right]$

Using MATLAB, we find the eigenvalues of $J_{1}$ as: 
$\lambda_{1}=-15.7860, \lambda_{2,3}=2.3930 \pm 18.8508 i$

Thus, the equilibrium $E_{1}$ is a saddle-focus, which is unstable.

The Jacobian matrix at $E_{2}$ is obtained as:

$J_{2}=J\left(E_{2}\right)=\left[\begin{array}{ccc}-10 & 190 & -10.6622 \\ 0 & 0 & 16.8819 \\ -0.8885 & -16.8819 & -1\end{array}\right]$

Using MATLAB, we find the eigenvalues of $J_{2}$ as:

$\lambda_{1}=-15.7860, \lambda_{2,3}=2.3930 \pm 18.8508 i$

Thus, the equilibrium $E_{2}$ is a saddle-focus, which is unstable. Hence, all the three equilibria of the system (1) are unstable equilibria.

\subsection{Lyapunov Exponents and Kaplan-Yorke Dimension}

For the chosen parameter values (2), the Lyapunov exponents of the novel chaotic system (1) are obtained using MATLAB as:

$L_{1}=2.71916, L_{2}=0, L_{3}=-13.72776$

Since the spectrum of Lyapunov exponents (20) has a positive term $L_{1}$, it follows that the 3-D novel system (1) is chaotic.

The maximal Lyapunov exponent (MLE) of the novel chaotic system (1) is $L_{1}=2.71916$.

The sum of the Lyapunov exponents is obtained as:

$L_{1}+L_{2}+L_{3}=-11.086<0$.

Thus, it follows that the novel chaotic system (1) is dissipative.

Also, the Kaplan-Yorke dimension of the novel chaotic system (1) is calculated as:

$D_{K Y}=2+\frac{L_{1}+L_{2}}{\left|L_{3}\right|}=2.19808$

Figure 5 depicts the dynamics of the Lyapunov exponents of the novel chaotic system (1).

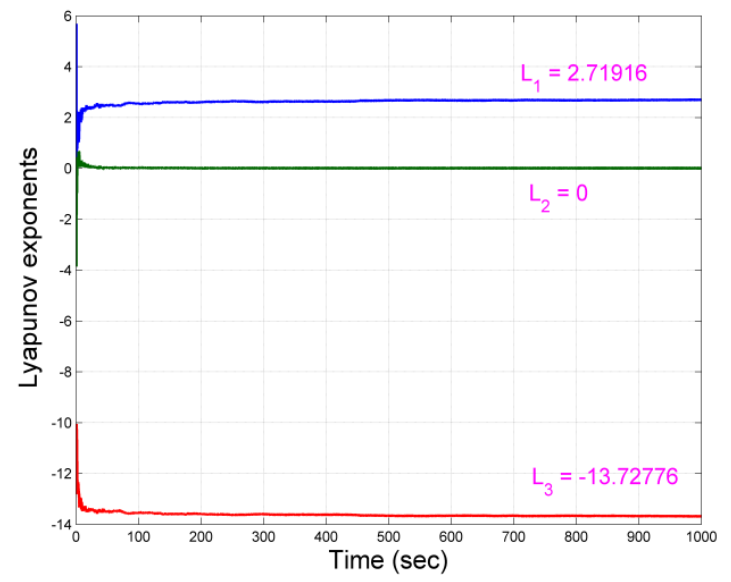

Fig. 5.Dynamics of the Lyapunov Exponents of the Novel System

\section{Adaptive Control of the Novel Chaotic System}

In this section, new results are derived for an adaptive controller to stabilize the novel chaotic system with unknown system parameters.

We consider the controlled novel 3-D chaotic system

$\left\{\begin{array}{l}\frac{d x_{1}}{d t}=a\left(x_{2}-x_{1}\right)+c x_{2} x_{3}+u_{1} \\ \frac{d x_{2}}{d t}=b x_{1}-x_{1} x_{3}+u_{2} \\ \frac{d x_{3}}{d t}=x_{1} x_{2}-x_{3}+u_{3}\end{array}\right.$

In (23), the parameters $a, b, c$ are unknown and $\mathrm{u}_{1}, \mathrm{u}_{2}, \mathrm{u}_{3}$ are adaptive controls to be determined using estimates $A(t)$, $B(t), C(t)$ for the unknown parameters $a, b, c$, respectively.

We consider the adaptive feedback control law

$$
\begin{aligned}
& u_{1}=-A(t)\left(x_{2}-x_{1}\right)-C(t) x_{2} x_{3}-k_{1} x_{1} \\
& u_{2}=-B(t) x_{1}+x_{1} x_{3}-k_{2} x_{2} \\
& u_{3}=-x_{1} x_{2}+x_{3}-k_{3} x_{3}
\end{aligned}
$$

In (24), $k_{1}, k_{2}, k_{3}$ are positive gain constants. Also, $A(t)$, $B(t)$ and $C(t)$ are estimates for the unknown system parameters $a, b, c$, respectively.

Substituting (24) into (23), we get

$$
\left\{\begin{aligned}
\frac{d x_{1}}{d t}= & (a-A(t))\left(x_{2}-x_{1}\right) \\
& +(c-C(t)) x_{2} x_{3}-k_{1} x_{1} \\
\frac{d x_{2}}{d t}= & (b-B(t)) x_{1}-k_{2} x_{2} \\
\frac{d x_{3}}{d t}= & -k_{3} x_{3}
\end{aligned}\right.
$$

The parameter estimation errors are defined by

$$
\left\{\begin{array}{l}
e_{a}(t)=a-A(t) \\
e_{b}(t)=b-B(t) \\
e_{c}(t)=c-C(t)
\end{array}\right.
$$

Using (26), we can simplify the dynamics (25) as

$$
\left\{\begin{array}{l}
\frac{d x_{1}}{d t}=e_{a}\left(x_{2}-x_{1}\right)+e_{c} x_{2} x_{3}-k_{1} x_{1} \\
\frac{d x_{2}}{d t}=e_{b} x_{1}-k_{2} x_{2} \\
\frac{d x_{3}}{d t}=-k_{3} x_{3}
\end{array}\right.
$$

Differentiating (26) with respect to $t$, we get

$$
\left\{\begin{array}{l}
\frac{d e_{a}}{d t}=-\frac{d A}{d t} \\
\frac{d e_{b}}{d t}=-\frac{d B}{d t} \\
\frac{d e_{c}}{d t}=-\frac{d C}{d t}
\end{array}\right.
$$

To derive an update law for the parameter estimates, we use adaptive control theory.

We consider the candidate Lyapunov function defined by

$$
V=\frac{1}{2}\left(x_{1}^{2}+x_{2}^{2}+x_{3}^{2}+e_{a}^{2}+e_{b}^{2}+e_{c}^{2}\right)
$$

Clearly, $V$ is a quadratic, positive definite function defined on $R^{6}$. 
Differentiating $V$ along the trajectories of (28) and (29), we obtain

$$
\begin{aligned}
\frac{d V}{d t}= & -k_{1} x_{1}^{2}-k_{2} x_{2}^{2}-k_{3} x_{3}^{2} \\
& +e_{a}\left[x_{1}\left(x_{2}-x_{1}\right)-\frac{d A}{d t}\right] \\
& +e_{b}\left[x_{1} x_{2}-\frac{d B}{d t}\right]+e_{c}\left[x_{1} x_{2} x_{3}-\frac{d C}{d t}\right]
\end{aligned}
$$

In view of (30), we take the parameter update law as:

$$
\left\{\begin{array}{l}
\frac{d A}{d t}=x_{1}\left(x_{2}-x_{1}\right) \\
\frac{d B}{d t}=x_{1} x_{2} \\
\frac{d C}{d t}=x_{1} x_{2} x_{3}
\end{array}\right.
$$

Theorem 1.The novel chaotic system (23) with unknown system parameters is globally and exponentially stabilized for all initial conditions by the adaptive control law (24) and the parameter update law (31), where $k_{1}, k_{2}, k_{3}$ are positive constants.

Proof. We prove this result using Lyapunov stability theory. For this purpose, we consider the quadratic Lyapunov function $V$ defined by (29), which is positive definite on $R^{6}$.

Substituting the parameter update law (31) into (30), we obtain the time derivative of $V$ as:

$\frac{d V}{d t}=-k_{1} x_{1}^{2}-k_{2} x_{2}^{2}-k_{3} x_{3}^{2}$

which is a negative semi-definite function on $R^{6}$.

Thus, we can conclude that the state vector $x(t)$ and the parameter estimation error are globally bounded, i.e. $\left[x_{1}(t), x_{2}(t), x_{3}(t), e_{a}(t), e_{b}(t), e_{c}(t)\right]^{T} \in L_{\infty}$.

We define $k=\min \left\{k_{1}, k_{2}, k_{3}\right\}$.

Then it follows from (32) that

$\frac{d V}{d t} \leq-k\|x\|^{2}$ or $k\|x\|^{2} \leq-\frac{d V}{d t}$

Integrating the inequality (33) from 0 to $t$, we get

$k \int_{0}^{t}\|x(\tau)\|^{2} d \tau \leq V(0)-V(t)$

From (34), it follows that $x(t) \in L_{2}$.

Using (27), we can conclude that $\dot{x} \in L_{\infty}$.

Thus, using Barbalat's lemma [135], we conclude that $x(t) \rightarrow 0$ exponentially as $t \rightarrow \infty$ for all initial conditions $x(0) \in R^{3}$.

This completes the proof.

For numerical simulations, the parameter values of the novel chaotic system (23) are taken as in the chaotic case, viz. $a=10, b=15$ and $c=12$. We take the gain constants as $k_{i}=5$ for $i=1,2,3$.

The initial values of the chaotic system (23) are taken as $x_{1}(0)=7.5, x_{2}(0)=5.3$ and $x_{3}(0)=-6.2$.

The initial values of the parameter estimates are taken as $A(0)=10.4, B(0)=6.3$ and $C(0)=2.5$.

Fig. 6 depicts the time-history of the controlled novel chaotic system. It is clear that the controlled system (23) is globally exponentially stable when the adaptive control law (24) and the parameter update law (31) are implemented.

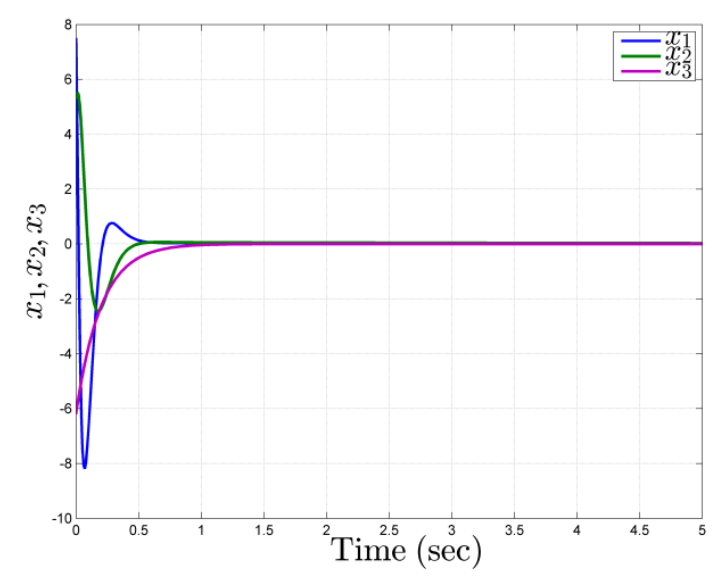

Fig. 6.Time-history of the controlled novel chaotic system.

\section{Adaptive Synchronization of the Identical Novel Chaotic Systems}

In this section, we derive new results for the adaptive synchronization of identical novel chaotic systems with unknown system parameters.

As the master system, we take the novel chaotic system

$\left\{\begin{array}{l}\frac{d x_{1}}{d t}=a\left(x_{2}-x_{1}\right)+c x_{2} x_{3} \\ \frac{d x_{2}}{d t}=b x_{1}-x_{1} x_{3} \\ \frac{d x_{3}}{d t}=x_{1} x_{2}-x_{3}\end{array}\right.$

where $x_{1}, x_{2}, x_{3}$ are state variables and $a, b, c$ are unknown, constant, parameters of the system.

As the slave system, we take the novel chaotic system

$\left\{\begin{array}{l}\frac{d y_{1}}{d t}=a\left(y_{2}-y_{1}\right)+c y_{2} y_{3}+u_{1} \\ \frac{d y_{2}}{d t}=b y_{1}-y_{1} y_{3}+u_{2} \\ \frac{d y_{3}}{d t}=y_{1} y_{2}-y_{3}+u_{3}\end{array}\right.$

where $y_{1}, y_{2}, y_{3}$ are state variables and $u_{1}, u_{2}, u_{3}$ are adaptive controllers to be designed.

The synchronization error between the identical chaotic systems is defined as:

$\left\{\begin{array}{l}e_{1}(t)=y_{1}(t)-x_{1}(t) \\ e_{2}(t)=y_{2}(t)-x_{2}(t) \\ e_{3}(t)=y_{3}(t)-x_{3}(t)\end{array}\right.$

The error dynamics is calculated as:

$\left\{\begin{array}{l}\frac{d e_{1}}{d t}=a\left(e_{2}-e_{1}\right)+c\left(y_{2} y_{3}-x_{2} x_{3}\right)+u_{1} \\ \frac{d e_{2}}{d t}=b e_{1}-y_{1} y_{3}+x_{1} x_{3}+u_{2} \\ \frac{d e_{3}}{d t}=-e_{3}+y_{1} y_{2}-x_{1} x_{2}+u_{3}\end{array}\right.$ 
We consider the adaptive control law

$$
\left\{\begin{aligned}
u_{1}= & -A(t)\left(e_{2}-e_{1}\right)-C(t)\left(y_{2} y_{3}-x_{2} x_{3}\right) \\
& -k_{1} e_{1} \\
u_{2}= & -B(t) e_{1}+y_{1} y_{3}-x_{1} x_{3}-k_{2} e_{2} \\
u_{3}= & e_{3}-y_{1} y_{2}+x_{1} x_{2}-k_{3} e_{3}
\end{aligned}\right.
$$

where $k_{1}, k_{2}, k_{3}$ are positive gains and $A(t), B(t), C(t)$ are estimates of the unknown parameters $a, b, c$, respectively.

The parameter estimation errors are defined by

$$
\left\{\begin{array}{l}
e_{a}(t)=a-A(t) \\
e_{b}(t)=b-B(t) \\
e_{c}(t)=c-C(t)
\end{array}\right.
$$

Substituting (39) into the error dynamics (38), we get

$$
\left\{\begin{aligned}
\frac{d e_{1}}{d t}= & (a-A(t))\left(e_{2}-e_{1}\right) \\
& +(c-C(t))\left(y_{2} y_{3}-x_{2} x_{3}\right)-k_{1} e_{1} \\
\frac{d e_{2}}{d t}= & (b-B(t)) e_{1}-k_{2} e_{2} \\
\frac{d e_{3}}{d t}= & -k_{3} e_{3}
\end{aligned}\right.
$$

Using (40), we can simplify the error dynamics (40) as:

$$
\left\{\begin{aligned}
\frac{d e_{1}}{d t}= & e_{a}\left(e_{2}-e_{1}\right) \\
& +e_{c}\left(y_{2} y_{3}-x_{2} x_{3}\right)-k_{1} e_{1} \\
\frac{d e_{2}}{d t}= & e_{b} e_{1}-k_{2} e_{2} \\
\frac{d e_{3}}{d t}= & -k_{3} e_{3}
\end{aligned}\right.
$$

Differentiating (40) with respect to $t$, we get

$$
\left\{\begin{array}{l}
\frac{d e_{a}}{d t}=-\frac{d A}{d t} \\
\frac{d e_{b}}{d t}=-\frac{d B}{d t} \\
\frac{d e_{c}}{d t}=-\frac{d C}{d t}
\end{array}\right.
$$

Next, we use Lyapunov stability theory for finding an update law for the parameter estimates.

Consider the quadratic Lyapunov function defined by

$$
V=\frac{1}{2}\left(e_{1}^{2}+e_{2}^{2}+e_{3}^{2}+e_{a}^{2}+e_{b}^{2}+e_{c}^{2}\right),
$$

which is positive definite on $R^{6}$.

Differentiating $V$ along the trajectories of (42) and (43), we get

$$
\begin{aligned}
\frac{d V}{d t}= & -k_{1} e_{1}^{2}-k_{2} e_{2}^{2}-k_{3} e_{3}^{2} \\
& +e_{a}\left[e_{1}\left(e_{2}-e_{1}\right)-\frac{d A}{d t}\right]+e_{b}\left[e_{1} e_{2}-\frac{d B}{d t}\right] \\
& +e_{c}\left[e_{1}\left(y_{2} y_{3}-x_{2} x_{3}\right)-\frac{d C}{d t}\right]
\end{aligned}
$$

In view of (45), we take the parameter update law as:

$$
\left\{\begin{array}{l}
\frac{d A}{d t}=e_{1}\left(e_{2}-e_{1}\right) \\
\frac{d B}{d t}=e_{1} e_{2} \\
\frac{d C}{d t}=e_{1}\left(y_{2} y_{3}-x_{2} x_{3}\right)
\end{array}\right.
$$

Theorem 2.The identical novel chaotic systems (35) and (36) with unknown system parameters are globally and exponentially synchronized for all initial conditions by the adaptive control law (39) and the parameter update law (46), where $k_{1}, k_{2}, k_{3}$ are positive constants.

Proof. We prove this result using Lyapunov stability theory.

For this purpose, we consider the quadratic Lyapunov function $V$ defined by (44), which is positive definite on $R^{6}$.

Substituting the parameter update law (46) into (45), we obtain the time derivative of $V$ as:

$\frac{d V}{d t}=-k_{1} e_{1}^{2}-k_{2} e_{2}^{2}-k_{3} e_{3}^{2}$,

which is a negative semi-definite function on $R^{6}$.

Thus, we can conclude that the synchronization error $e(t)$ and the parameter estimation error are globally bounded.

We define $k=\min \left\{k_{1}, k_{2}, \underline{\mathrm{k}}_{3}\right\}$. Then we get

$\frac{d V}{d t} \leq-k\|e\|^{2}$ or $k\|e\|^{2} \leq-\frac{d V}{d t}$

Integrating the inequality (48) from 0 to $t$, we get

$k \int_{0}^{t}\|e(\tau)\|^{2} d \tau \leq V(0)-V(t)$

From (49), it follows that $e(t) \in L_{2}$. Using (42), we can conclude that $\dot{x} \in L_{\infty}$.

Thus, using Barbalat's lemma [135], we conclude that $e(t) \rightarrow 0$ exponentially as $t \rightarrow \infty$ for all initial conditions $e(0) \in R^{3}$.This completes the proof.

For numerical simulations, the parameter values of the novel chaotic systems (35) and (36) are taken as in the chaotic case, viz. $a=10, b=15$ and $c=12$. We take the gain constants as $k_{i}=5$ for $i=1,2,3$.

The initial conditions of the master system (35) are taken as $x_{1}(0)=5.2, x_{2}(0)=8.3$ and $x_{3}(0)=-2.7$.

The initial conditions of the slave system (36) are taken as $y_{1}(0)=-3.5, y_{2}(0)=4.6$ and $y_{3}(0)=5.4$.

The initial conditions of the parameter estimates are taken as $A(0)=2.1, B(0)=9.4$ and $C(0)=3.8$.

Figures 7-9 describe the complete synchronization of the novel chaotic systems (35) and (36), while Fig. 10 describes the time-history of the synchronization errors $e_{1}, e_{2}, e_{3}$. 


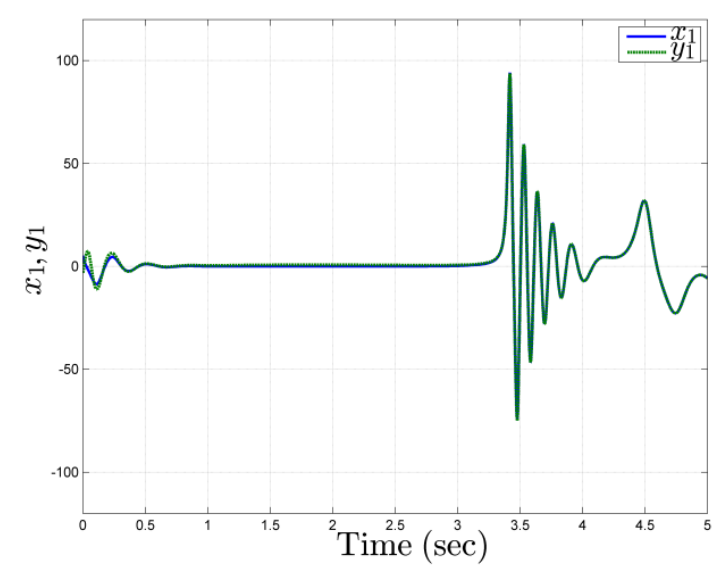

Fig. 7.Synchronization of the states $x_{1}$ and $y_{1}$ of the chaotic systems.

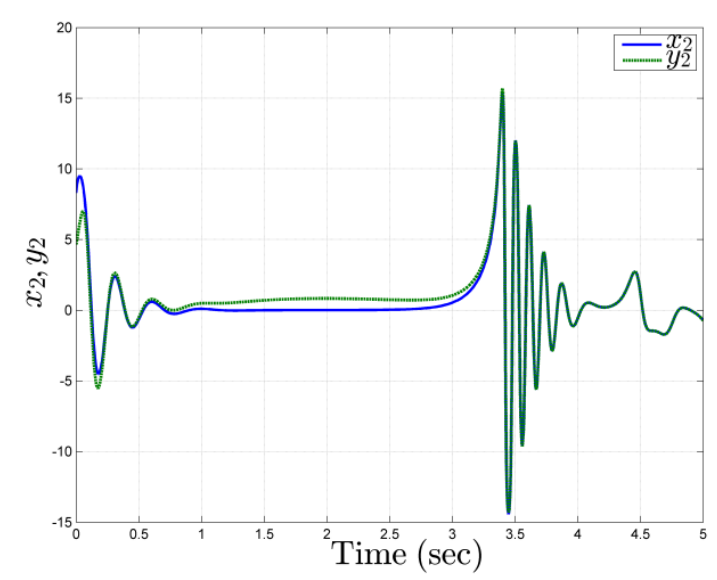

Fig. 8.Synchronization of the states $x_{2}$ and $y_{2}$ of the chaotic systems.

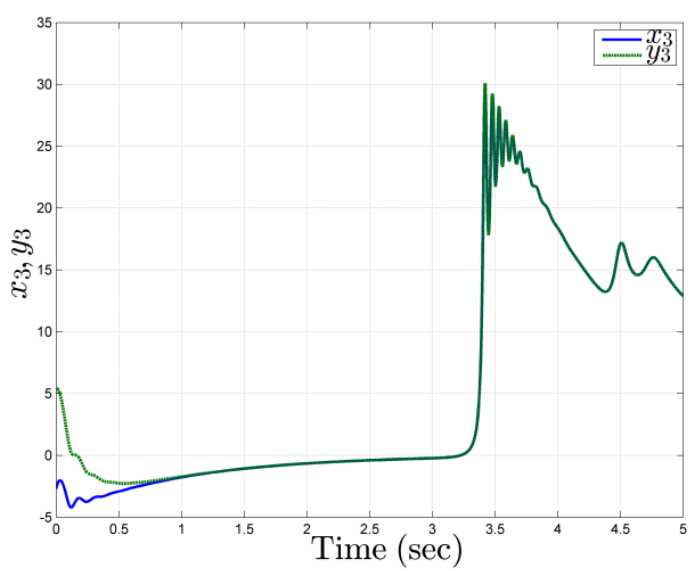

Fig. 9.Synchronization of the states $x_{3}$ and $y_{3}$ of the chaotic systems.

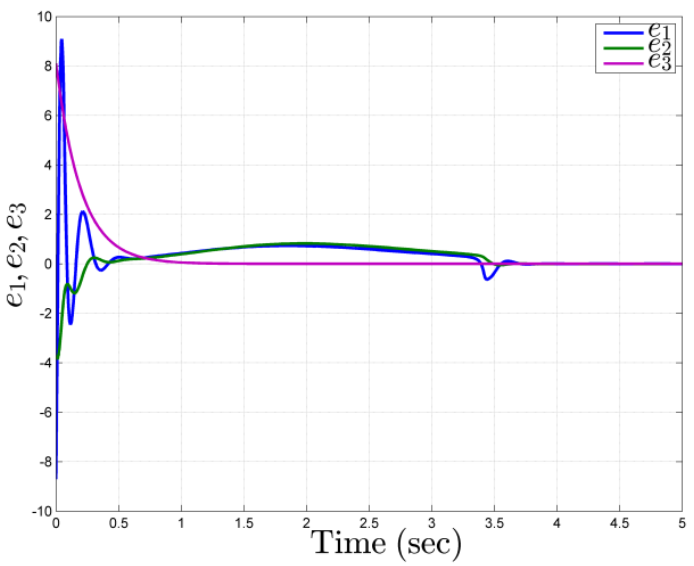

Fig. 10.Time-history of the synchronization errors.

\section{LabVIEW Implementation of the 3-D Novel Chaotic system and its Adaptive Synchronization}

The proposed 3-D novel chaotic system is then implemented in LabVIEW. The state equations of the new chaotic system are implemented in CSD tool book of LabVIEW. Fixed step simulation method is applied for the iteration process. The configuration parameters are altered to match the simulation environment. Simulation time waveform converters are deployed to generate time based signals for waveform plotting. Reshape array blocks are used to get 1D array elements from double precision data. The LabVIEW VI model is shown in Fig. 11. The time waveforms and phase portraits of the master system are shown in Figs. 12 \& 13 respectively. The designed controller for the adaptive synchronization is shown in Fig. 14. The errors plots of the synchronized systems are shown in

Fig. 15.

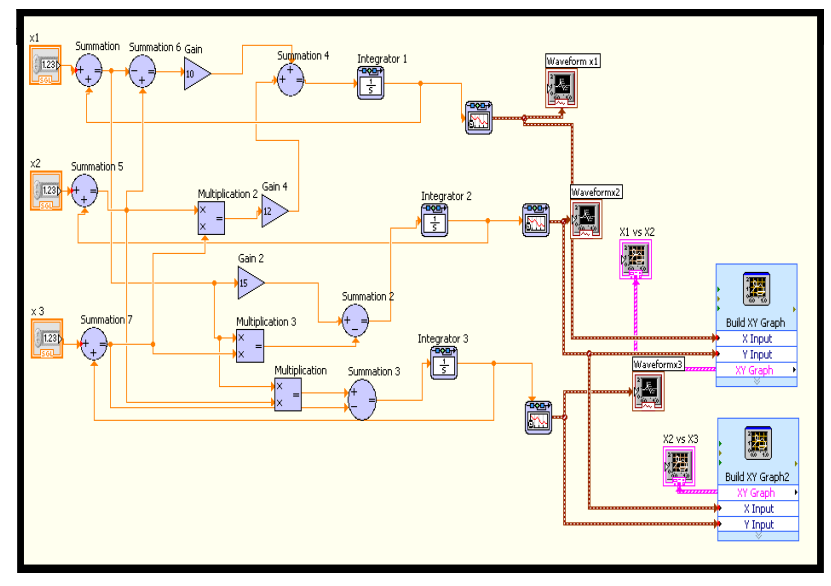

Fig. 11. VI Model for the new chaotic system. 

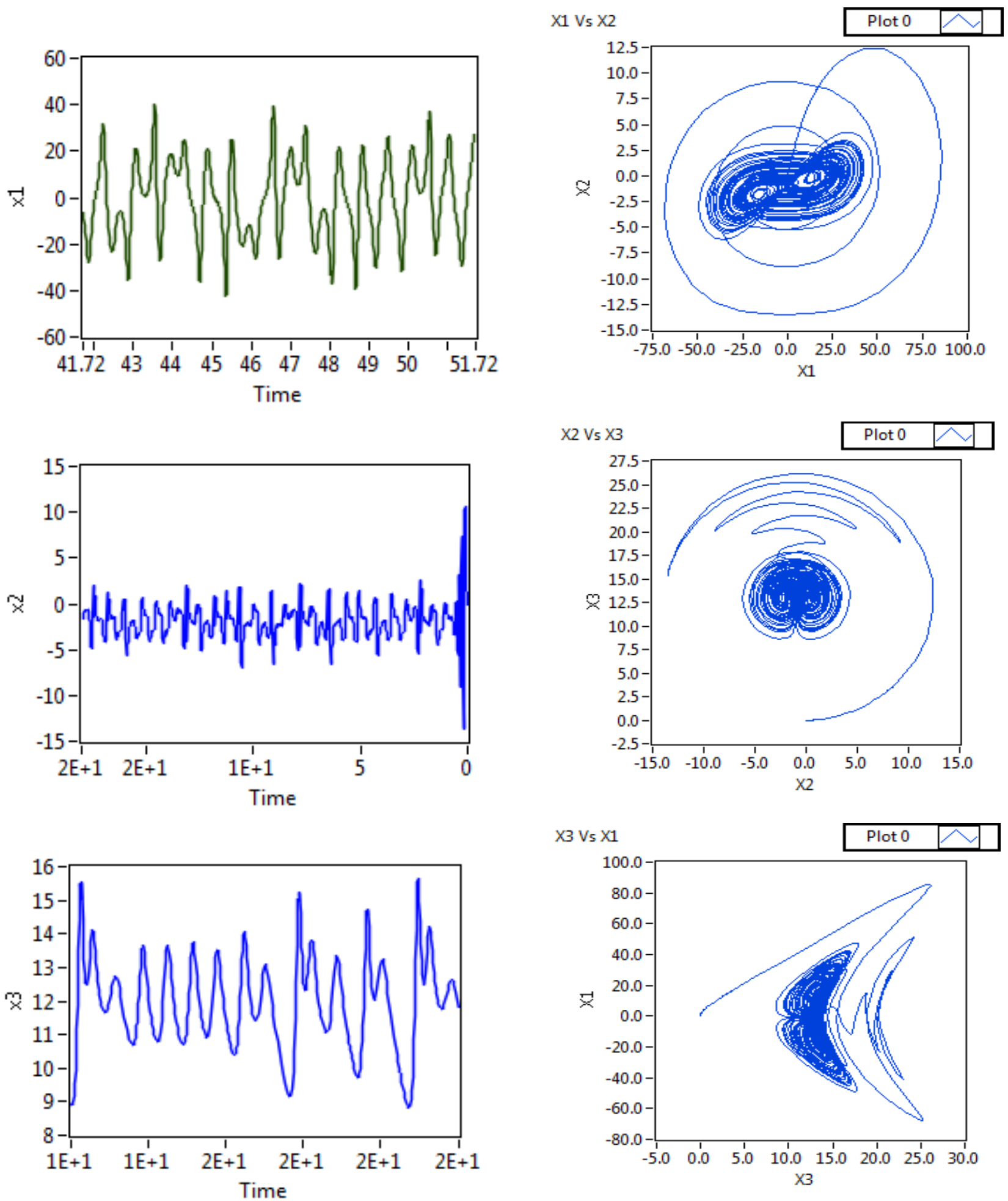

Fig. 12. Time waveforms of the state variables.

Fig. 13. 2-D Phase Portraits of the novel chaotic attractor. 


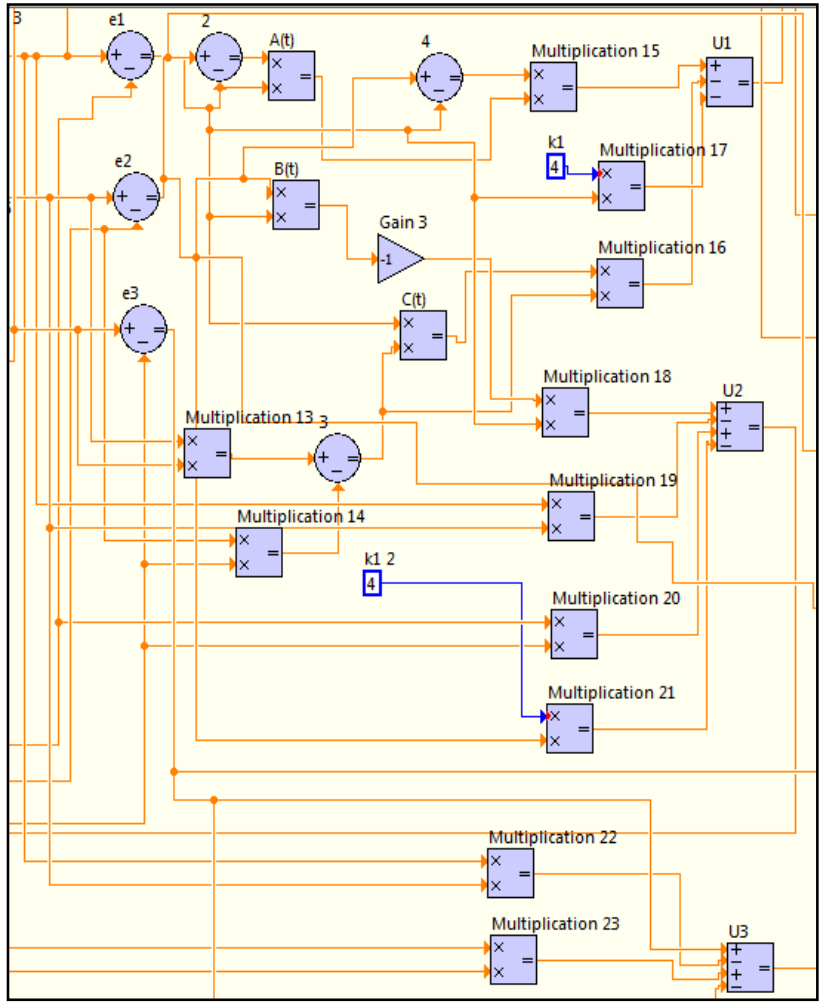

Fig. 14. The controller $u$ implemented in LabVIEW.

\section{Conclusion}

In this work a seven-term novel 3-D chaotic system with three quadratic terms was presented. The fundamental properties of the system such as dissipativity, symmetry, equilibria, Lyapunov exponents and Kaplan-Yorke dimension as well as its phase portraits were described in detail. Also, an adaptive controller for stabilizing the proposed system with unknown parameters was designed. Furthermore, the case of chaos synchronization, of two identical chaotic systems of this type, by using an adaptive controller was studied. Finally, the LabVIEW implementation of the novel chaotic system was presented for confirming the feasibility of the theoretical chaotic system.
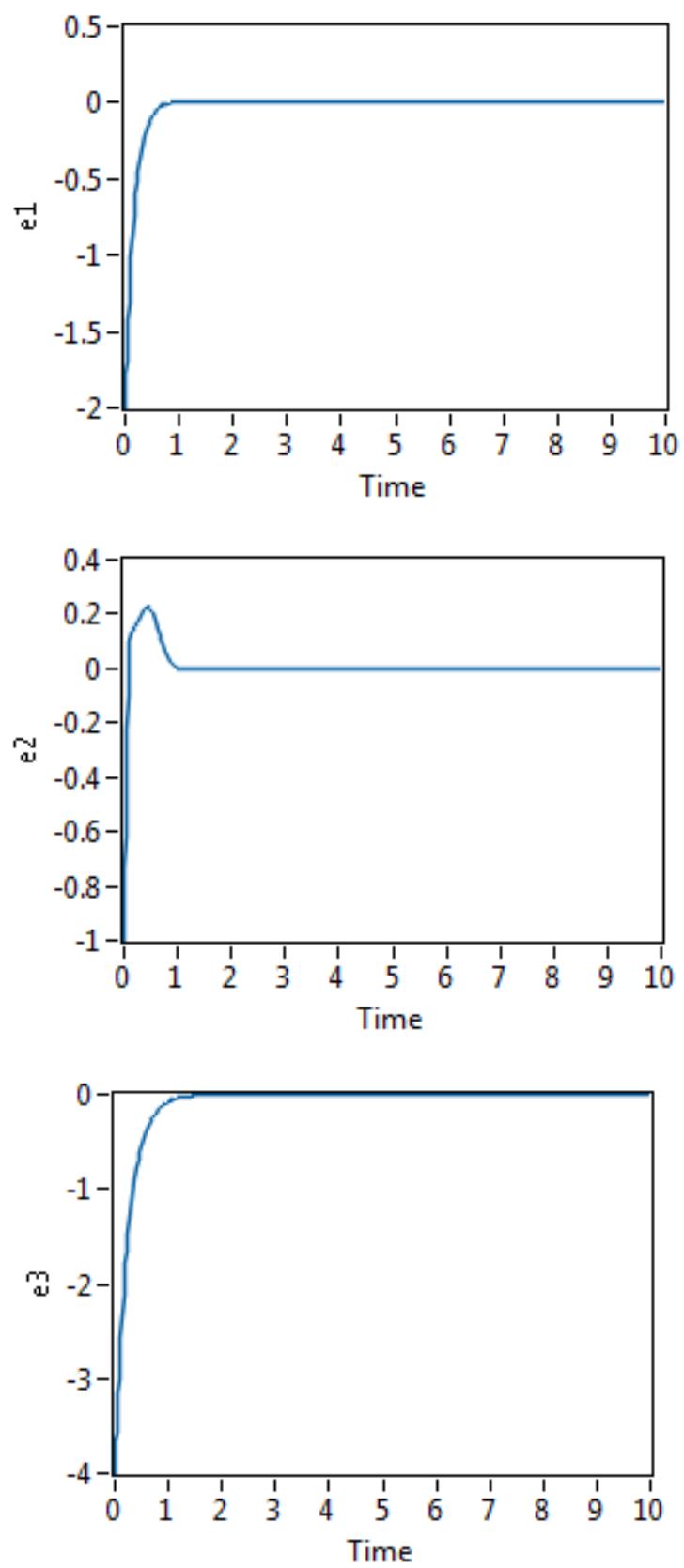

Fig. 15. Time-history of the synchronization errors $e_{1}, e_{2}, e_{3}$.

\section{References}

1. S.H. Strogatz, Nonlinear dynamics and chaos: With applications to physics, biology, chemistry, and engineering, Perseus Books, Massachusetts, US (1994).

2. E.N. Lorenz, Deterministic nonperiodic flow, Journal of the Atmospheric Sciences, vol. 20, pp. 130-141 (1963).

3. O.E. Rössler, An equation for continuous chaos, Physics Letters A, vol. 57, pp. 397-398 (1976).

4. M.I. Rabinovich and A.L. Fabrikant, Stochastic self-modulation of waves in nonequilibrium media, Sov. Phys. JETP, vol. 50, pp. 311-317, (1979)

5. A. Arneodo, P. Coullet, and C. Tresser, Possible new strange attractors with spiral structure, Communications in Mathematical Physics, vol. 79, pp. 573-579 (1981).

6. J.C. Sprott, Some simple chaotic flows, Physical Review E, vol. 50, pp. 647-650 (1994).
7. G. Chen and T. Ueta, Yet another chaotic oscillator, International Journal of Bifurcation and Chaos, vol. 9, pp. 14651466 (1999).

8. J. Lü and G. Chen, A new chaotic attractor coined, International Journal of Bifurcation and Chaos, vol. 12, pp. 659-661 (2002).

9. R. Shaw, Strange attractors, chaotic behavior and information flow, Zeitschrift für Naturforschung, vol. 36, pp. 80-112 (1981).

10. B. Feeny and F.C. Moon, Chaos in a forced dry-friction oscillator: Experiments and numerical modeling, Journal of Sound and Vibration, vol. 170, pp. 303-323 (1994).

11. T. Shimizu and N. Moroika, On the bifurcation of a symmetric limit cycle to an asymmetric one in a simple model, Physics Letters A, vol. 76, pp. 201-204 (1980).

12. W. Liu and G. Chen, A new chaotic system and its generation, International Journal of Bifurcation and Chaos, vol. 13, pp. 261267 (2003) 
13. G. Cai and Z. Tan, Chaos synchronization of a new chaotic system via nonlinear control, Journal of Uncertain Systems, vol. 1 , pp. 235-240 (2007).

14. G. Tigan and D. Opris, Analysis of a 3D chaotic system, Chaos, Solitons and Fractals, vol. 36, pp. 1315-1319 (2008).

15. G.P. Kennedy, Chaos in the Colpitts oscillator, IEEE Transactions on Circuits and Systems-I, vol. 41, pp. 771-774 (1994).

16. J. Wang, D. Lu and L. Tian, Global synchronization for time delay of WINDMI system, Chaos, Solitons and Fractals, vol. 30, pp. 629-635 (2006).

17. W. Zhou, Y. Xu, H. Lu, and L. Pan, On dynamics analysis of a new chaotic attractor, Physics LettersA, vol. 372, pp. 5773-5777 (2008).

18. D. Li, A three-scroll chaotic attractor, Physics Letters A, vol. 372, pp. 387-393 (2008)

19. Z. Elhadj, Dynamical analysis of a 3-D chaotic system with only two quadratic nonlinearities, Journal of Systems Science and Complexity, vol. 21, pp. 67-75 (2008).

20. L. Pan, D. Xuand W. Zhou, Controlling a novel chaotic attractor using linear feedback, Journal of Information and Computing Science, vol. 5, pp. 117-124 (2010).

21. V. Sundarapandian, Analysis and anti-synchronization of a novel chaotic system via active and adaptive controllers, Journal of Engineering Science and Technology Review, vol. 6, pp. 45-52 (2013).

22. F. Yu, C. Wang, Q. Wan, and Y. Hu, Complete switched modified function projective synchronization of a five-term chaotic system with uncertain parameters and disturbances, Pramana, vol. 80, pp. 223-235 (2013).

23. V. Sundarapandian and I. Pehlivan, Analysis, control, synchronization and circuit design of a novel chaotic system, Mathematical and Computer Modelling, vol. 55, pp. 1904-1915 (2012).

24. C. Zhu, Y. Liu, and Y. Guo, Theoretical and numerical study of a new chaotic system, Intelligent Information Management, vol. 2, pp. 104-109 (2010)

25. S. Vaidyanathan, A new six-term 3-D chaotic system with an exponential nonlinearity, Far East Journal of Mathematical Sciences, vol. 79, pp. 135-143 (2013).

26. S. Vaidyanathan, Analysis and adaptive synchronization of two novel chaotic systems with hyperbolic sinusoidal and cosinusoidal nonlinearity and unknown parameters, Journal of Engineering Science and Technology Review, vol. 6, pp. 53-65 (2013).

27. S. Vaidyanathan, A new eight-term 3-D polynomial chaotic system with three quadratic nonlinearities, Far East Journal of Mathematical Sciences, vol. 84, pp. 219-226 (2014).

28. S. Vaidyanathan, Analysis, control and synchronization of a sixterm novel chaotic system with three quadratic nonlinearities, International Journal of Modelling, Identification and Control, vol. 22, pp. 41-53 (2014).

29. S. Vaidyanathan, Analysis and adaptive synchronization of eight-term 3-D polynomial chaotic systems with three quadratic nonlinearities, European Physical Journal: Special Topics, vol. 223, pp. 1519-1529 (2014).

30. S. Vaidyanathan, Ch. Volos, V.T. Pham, K. Madhavan, and B.A. Idowu, Adaptive backstepping control, synchronization and circuit simulation of a 3-D novel jerk chaotic system with two hyperbolic sinusoidal nonlinearities, Archives of Control Sciences, vol. 24(3), pp. 257-285 (2014).

31. S. Vaidyanathan, Generalized projective synchronisation of novel 3-D chaotic systems with an exponential nonlinearity via active and adaptive control, International Journal of Modelling, Identification and Control, vol. 22 (3), pp. 207-217 (2014).

32. S. Vaidyanathan and K. Madhavan, Analysis, adaptive control and synchronization of a seven-term novel 3-D chaotic system, International Journal of Control Theory and Applications, vol. 6, pp. 121-137 (2013)

33. I. Pehlivan, I.M. Moroz, and S. Vaidyanathan, Analysis, synchronization and circuit design of a novel butterfly attractor, Journal of Sound and Vibration, vol. 333, pp. 5077-5096 (2014).

34. S. Jafari and J.C. Sprott, Simple chaotic flows with aline equilibrium, Chaos, Solitons and Fractals, vol. 57, pp. 79-84, 2013.

35. V.T. Pham, C. Volos, S. Jafari, Z. Weiand X. Wang, Constructing a novel no-equilibrium chaotic system,
International Journal of Bifurcation and Chaos, vol. 24, 1450073 (2014).

36. H.B. Fotsin and J. Daafouz, Adaptive synchronization of uncertain Colpitts oscillators based on parameter identification, Physics Letters A, vol. 339, pp. 304-315 (2005).

37. G.H. Li, S.P. Zhou and K. Yang, Controlling chaos in Colpitts oscillator, Chaos, Solitons and Fractals, vol. 33, pp. 582-587 (2007).

38. A. Sharma, V. Patidar, G. Purohit, and K.K. Sud, Effects on the bifurcation and chaos in forced Duffing oscillator due to nonlinear damping, Communications in Nonlinear Science and Numerical Simulation, vol. 17, pp. 2254-2269 (2012).

39. S. Donati and S.K. Hwang, Chaos and high-level dynamics in coupled lasers and their applications, Progress in Quantum Electronics, vol. 36, pp. 293-341 (2012).

40. N. Li, W. Pan, L. Yan, B. Luo, and X. Zou, Enhanced chaos synchronization and communication in cascade-coupled semiconductor ring lasers, Communications in Nonlinear Science and Numerical Simulation, vol. 19, pp. 1874-1883 (2014).

41. U. Nehmzow and K. Walker, Quantitative description of robotenvironment interaction using chaos theory, Robotics and Autonomous Systems, vol. 53, pp. 177-193 (2005).

42. S. Iqbal, X. Zang, Y. Zhu, and J. Zhao, Bifurcations and chaos in passive dynamic walking: A review, Robotics and Autonomous Systems, vol. 62, pp. 889-909 (2014).

43. Ch.K. Volos, I.M. Kyprianidis, and I.N. Stouboulos, A chaotic path planning generator for autonomous mobile robots, Robotics and Autonomous Systems, vol. 60, pp. 651-656, (2012).

44. Ch.K. Volos, I.M. Kyprianidis, and I.N. Stouboulos, Experimental investigation on coverage performance of a chaotic autonomous mobile robot, Robotics and Autonomous Systems, vol. 61(12), pp. 1314-1322(2013).

45. J.C. Roux, Chaos in experimental chemical systems: two examples, North-Holland Mathematics Studies, vol. 103, pp. 345-352 (1985).

46. Y.N. Li, L. Chen, Z.S. Cai, and X.Z. Zhao, Study on chaos synchronization in the Belousov-Zhabotinsky chemical system, Chaos, Solitons and Fractals, vol. 17, pp. 699-707 (2003).

47. M. Kyriazis, Applications of chaos theory to the molecular biology of aging, Experimental Gerontology, vol. 26, pp. 569572 (1991).

48. G. Böhm, Protein folding and deterministic chaos: Limits of protein folding simulations and calculations, Chaos, Solitons and Fractals, vol. 1, pp. 375-382 (1991).

49. J.C. Sprott, J.A. Vano, J.C. Wildenberg, M.B. Anderson, and J.K. Noel, Coexistence and chaos in complex ecologies, Physics Letters A, vol. 335, pp. 207-212 (2005).

50. B. Sahoo and S. Poria, The chaos and control of a food chain model supplying additional food to top-predator, Chaos, Solitons and Fractals, vol. 58, pp. 52-64 (2014).

51. T.A. Denton, G.A. Diamond, R.H. Helfant, S. Khan, and H. Karagueuzian, Fascinating rhythm: A primer on chaos theory and its applications to cardiology, American Heart Journal, vol. 120, pp. 1419-1440 (1990).

52. A. Wu, S. Wen, and Z. Zeng, Synchronization control of a class of memristor-based neural networks, Information Sciences, vol. 183, pp. 106-116, (2012).

53. S. Wen, Z. Zeng, and T. Huang, Adaptive synchronization of memristor-based Chua's circuits, Physics Letters A, vol. 376, pp. 2775-2780, (2012).

54. A. Wu, and Z. Zeng, Anti-synchronization control of a class of memristive recurrent neural networks, Communications in Nonlinear Science and Numerical Simulation, vol. 18, pp. 373385, (2013).

55. G. He, Z. Cao, P. Zhu, and H. Ogura, Controlling chaos in a chaotic neural network, Neural Networks, vol. 16, pp. 11951200 (2003).

56. E. Kaslik and S. Sivasundaram, Nonlinear dynamics and chaos in fractional-order neural networks, Neural Networks, vol. 32, pp. 245-256 (2012).

57. I.M. Kyprianidis and A.T. Makri, Complex dynamics of FitzHugh-Nagumo type neurons coupled with gap junction under external voltage stimulation, Journal of Engineering Science and Technology Review, vol. 6(4), pp. 104-114(2013).

58. K. Suzuki and Y. Imai, Decryption characteristics in message modulation type chaos secure communication system using 
optical fiber ring resonators, Optics Communications, vol. 259, pp. 88-93 (2006).

59. X.Y. Wang and Y.F. Gao, A switch-modulated method for chaos digital secure communication based on user-defined protocol, Communications in Nonlinear Science and Numerical Simulation, vol. 15, pp. 99-104 (2010).

60. O.I. Moskalenko, A.A. Koronovskii, and A.E. Hramov, Generalized synchronization of chaos for secure communication: Remarkable stability to noise, Physics Letters A, vol. 374, pp. 2925-2931 (2010).

61. A. Abdullah, Synchronization and secure communication of uncertain chaotic systems based on full-order and reduced-order output-affine observers, Applied Mathematics and Computation, vol. 219, pp. 10000-10011 (2013).

62. N. Smaoui and A. Kanso, Cryptography with chaos and shadowing, Chaos, Solitons and Fractals, vol. 42, pp. 2312-2321 (2009).

63. R. Rhouma and S. Belghith, Cryptanalysis of a chaos-based cryptosystem on DSP, Communications in Nonlinear Science and Numerical Simulation, vol. 16, pp. 876-884 (2011).

64. Ch K. Volos, I.M. Kyprianidis, and I.N. Stouboulos, Text encryption scheme realized with a chaotic pseudo-random bit generator, Journal of Engineering Science and Technology Review, vol. 6(4), pp. 9-14 (2013).

65. Ch.K. Volos, I.M. Kyprianidis, and I.N. Stouboulos, Image encryption process based on chaotic synchronization phenomena, Signal Processing, vol. 93(5), pp. 1328-1340 (2013).

66. D. Guégan, Chaos in economics and finance, Annual Reviews in Control, vol. 33, pp. 89-93 (2009).

67. Ch.K. Volos, I.M. Kyprianidis, and I.N. Stouboulos, Synchronization phenomena in coupled nonlinear systems applied in economic cycles, WSEAS Trans. Systems, vol. 11(12), pp. 681-690(2012).

68. P. Caraiani, Testing for nonlinearity and chaos in economic time series with noise titration, Economics Letters, vol. 120, pp. 192194 (2013)

69. V. Sundarapandian, Output regulation of the Lorenz attractor, Far East Journal of Mathematical Sciences, vol. 42, pp. 289-299 (2010).

70. S. Vaidyanathan, Output regulation of Arneodo-Coullet chaotic system, Communications in Computer and Information Science, vol. 131, pp. 585-593 (2011).

71. S. Vaidyanathan, Output regulation of the unified chaotic system, Communications in Computer and Information Science, vol. 198, pp. 1-9 (2011)

72. S. Vaidyanathan, Output regulation of the Liu chaotic system, Applied Mechanics and Materials, vols. 110-116, pp. 3982-3989 (2012).

73. G. Chen, A simple adaptive feedback control method for chaos and hyper-chaos control, Applied Mathematics and Computation, vol. 217, pp. 7258-7264 (2011).

74. J. Zheng, A simple universal adaptive feedback controller for chaos and hyperchaos control, Computers \& Mathematics with Applications, vol. 61, pp. 2000-2004 (2011).

75. S. Vaidyanathan, Adaptive controller and synchronizer design for the Qi-Chen chaotic system, Lecture Notes of the Institute for Computer Sciences, Social-Informatics and Telecommunications Engineering, vol. 85, pp. 124-133 (2012).

76. V. Sundarapandian, Adaptive control and synchronization design for the Lu-Xiao chaotic system, Lecture Notes in Electrical Engineering, vol. 131, pp. 319-327 (2013).

77. S. Vaidyanathan, A ten-term novel 4-D hyperchaotic system with three quadratic nonlinearities and its control, International Journal of Control Theory and Applications, vol. 6, pp. 97-109 (2013).

78. S. Vaidyanathan, Analysis, control and synchronization of hyperchaotic Zhou system via adaptive control, Advances in Intelligent Systems and Computing, vol. 177, pp. 1-10 (2013).

79. D. Yang and J. Zhou, Connections among several chaos feedback control approaches and chaotic vibration control of mechanical systems, Communications in Nonlinear Science and Numerical Simulation, vol. 19, pp. 3954-3968 (2014).

80. M.T. Yassen, Chaos control of chaotic dynamical systems using backstepping design, Chaos, Solitons and Fractals, vol. 27, pp. 537-548 (2006).

81. J.A. Laoye, U.E. Vincent, and S.O. Kareem, Chaos control of 4D chaotic systems using recursive backstepping nonlinear controller, Chaos, Solitons and Fractals, vol. 39, pp. 356-362 (2009).

82. D. Lin, X. Wang, F. Nian, and Y. Zhang, Dynamic fuzzy neural networks modeling and adaptive backstepping tracking control of uncertain chaotic systems, Neurocomputing, vol. 73, pp. 2873-2881 (2010).

83. S. Vaidyanathan, Sliding mode control based global chaos control of Liu-Liu-Liu-Su chaotic system, International Journal of Control Theory and Applications, vol. 5, pp. 15-20 (2012).

84. S. Vaidyanathan, Global chaos control of hyperchaotic Liu system via sliding mode control, vol. 5, pp. 117-123 (2012).

85. L. Kocarev and U. Parlitz, General approach for chaos synchronization with applications to communications, Physical Review Letters, vol. 74, pp. 5028-5030 (1995).

86. K. Murali and M. Lakshmanan, Secure communication using a compound signal using sampled-data feedback, Applied Mathematics and Mechanics, vol. 11, pp. 1309-1315, (1995).

87. M. Feki, An adaptive chaos synchronization scheme applied to secure communication, Chaos, Solitons and Fractals, vol. 18, pp. 141-148 (2003).

88. J. Yang and F. Zhu, Synchronization for chaotic systems and chaos-based secure communications via both reduced-order and step-by-step sliding mode observers, Communications in Nonlinear Science and Numerical Simulation, vol. 18, pp. 926937 (2013).

89. L. Kocarev, Chaos-based cryptography: a brief overview, IEEE Circuits and Systems, vol. 1, pp. 6-21 (2001).

90. H. Gao, Y. Zhang, S. Liang and D. Li, A new chaotic algorithm for image encryption, Chaos, Solitons and Fractals, vol. 29, pp. 393-399 (2006).

91. Y. Wang, K.W. Wang, X. Liao and G. Chen, A new chaos-based fast image encryption, Applied Soft Computing, vol. 11, pp. 514-522, (2011).

92. L.M. Pecora and T.L. Carroll, Synchronization in chaotic systems, vol. 64, pp. 821-825 (1990).

93. S. Vaidyanathan and S. Rasappan, New results on the global chaos synchronization for Liu-Chen-Liu and Lü chaotic systems, Communications in Computer and Information Science, vol. 102, pp. 20-27 (2010).

94. S. Vaidyanathan and S. Rasappan, Hybrid synchronization of hyperchaotic Qi and Lü systems by nonlinear control, Communications in Computer and Information Science, vol. 131, pp. 585-593 (2011).

95. S. Vaidyanathan and K. Rajagopal, Anti-synchronization of Li and $\mathrm{T}$ chaotic systems by active nonlinear control, Communications in Computer and Information Science, vol. 198, pp. 175-184 (2011).

96. S. Vaidyanathan and S. Rasappan, Global chaos synchronization of hyperchaotic Bao and Xu systems by active nonlinear control, Communications in Computer and Information Science, vol. 198, pp. 10-17 (2011).

97. S. Vaidyanathan and K. Rajagopal, Global chaos synchronization of hyperchaotic Pang and Wang systems by active nonlinear control, Communications in Computer and Information Science, vol. 204, pp. 84-93 (2011).

98. S. Vaidyanathan, Hybrid chaos synchronization of Liu and Lü systems by active nonlinear control, Communications in Computer and Information Science, vol. 204, pp. 1-10 (2011).

99. P. Sarasu and V. Sundarapandian, Active controller design for generalized projective synchronization of four-scroll chaotic systems, International Journal of Systems Signal Control and Engineering Application, vol. 4, pp. 26-33 (2011).

100. S. Vaidyanathan and K. Rajagopal, Hybrid synchronization of hyperchaotic Wang-Chen and hyperchaotic Lorenz systems by active non-linear control, International Journal of Systems Signal Control and Engineering Application, vol. 4, pp. 55-61 (2011).

101. S. Pakiriswamy and S. Vaidyanathan, Generalized projective synchronization of three-scroll chaotic systems via active control, Lecture Notes of the Institute for Computer Sciences, Social-Informatics and Telecommunications Engineering, vol. 85, pp. 146-155 (2012).

102. V. Sundarapandian and R. Karthikeyan, Hybrid synchronization of hyperchaotic Lorenz and hyperchaotic Chen systems via active control, Journal of Engineering and Applied Sciences, vol. 7, pp. 254-264 (2012). 
103. R. Karthikeyan and V. Sundarapandian, Hybrid chaos synchronization of four-scroll systems via active control, Journal of Electrical Engineering, vol. 65, pp. 97-103 (2014).

104. E.M. Shahverdiev and K.A. Shore, Impact of modulated multiple optical feedback time delays on laser diode chaos synchronization, Optics Communications, vol. 282, pp. 35683572 (2009).

105. T. Botmart, P. Niamsup, and X. Liu, Synchronization of nonautonomous chaotic systems with time-varying delay via delayed feedback control, Communications in Nonlinear Science and Numerical Simulation, vol. 17, pp. 1894-1907 (2012).

106. S. Bowong, Adaptive synchronization between two different chaotic dynamical systems, Communications in Nonlinear Science and Numerical Simulation, vol. 12, pp. 976-985 (2007).

107. W. Lin, Adaptive chaos control and synchronization in only locally Lipschitz systems, Physics Letters A, vol. 372, pp. 31953200 (2008).

108. H. Salarieh and A. Alasty, Adaptive chaos synchronization in Chua's systems with noisy parameters, Mathematics and Computers in Simulation, vol. 79, pp. 233-241 (2008).

109. H. Salarieh and A. Alasty, Adaptive synchronization of two chaotic systems with stochastic unknown parameters, Communications in Nonlinear Science and Numerical Simulation, vol. 14, pp. 508-519 (2009).

110. S. Vaidyanathan and K. Rajagopal, Global chaos synchronization of Lü and Pan systems by adaptive nonlinear control, Communications in Computer and Information Science, vol. 205, pp. 193-202 (2011).

111. V. Sundarapandian and R. Karthikeyan, Anti-synchronization of Lü and Pan chaotic systems by adaptive nonlinear control, European Journal of Scientific Research, vol. 64, pp. 94-106 (2011).

112. V. Sundarapandian and R. Karthikeyan, Anti-synchronization of hyperchaotic Lorenz and hyperchaotic Chen systems by adaptive control, International Journal of Systems Signal Control and Engineering Application, vol. 4, pp. 18-25 (2011).

113. V. Sundarapandian and R. Karthikeyan, Adaptive antisynchronization of uncertain Tigan and Li systems, Journal of Engineering and Applied Sciences, vol. 7, pp. 45-52 (2012).

114. P. Sarasu and V. Sundarapandian, Generalized projective synchronization of three-scroll chaotic systems via adaptive control, European Journal of Scientific Research, vol. 72, pp. 504-522 (2012).

115. P. Sarasu and V. Sundarapandian, Generalized projective synchronization of two-scroll systems via adaptive control, International Journal of Soft Computing, vol. 7, pp. 146-156 (2012).

116. P. Sarasu and V. Sundarapandian, Adaptive controller design for the generalized projective synchronization of 4-scroll systems, International Journal of Systems Signal Control and Engineering Application, vol. 5, pp. 21-30 (2012).

117. S. Vaidyanathan and K. Rajagopal, Global chaos synchronization of hyperchaotic Pang and hyperchaotic Wang systems via adaptive control, International Journal of Soft Computing, vol. 7, pp. 28-37 (2012).

118. S.H. Lee, V. Kapila, M. Porfiri, and A. Panda, Master-slave synchronization of continuously and intermittently coupled sampled-data chaotic oscillators, Communications in Nonlinear Science and Numerical Simulation, vol. 15, pp. 4100-4113 (2010).

119. X.Z. Jin and J.H. Park, Adaptive synchronization for a class of faulty and sampling coupled networks with its circuit implement, Journal of the Franklin Institute, vol. 351, pp. 4317-4333 (2014).
120. C.K. Zhang, L. Jiang, Y. He, Q.H. Wu and M. Wu, Asymptotical synchronization for chaotic Lur'e systems using sampled-data control, Communications in Nonlinear Science and Numerical Simulation, vol. 18, pp. 2743-2751 (2013).

121. X. Xiao, L. Zhou, and Z. Zhang, Synchronization of chaotic Lur'e systems with quantized sampled-data controller, Communications in Nonlinear Science and Numerical Simulation, vol. 19, pp. 2039-2047 (2014).

122. S. Rasappan and S. Vaidyanathan, Global chaos synchronization of WINDMI and Coullet chaotic systems by backstepping control, Far East Journal of Mathematical Sciences, vol. 67, pp. 265-287 (2012).

123. S. Rasappan and S. Vaidyanathan, Synchronization of hyperchaotic Liu system via backstepping control with recursive feedback, Communications in Computer and Information Science, vol. 305, pp. 212-221 (2012).

124. S. Rasappan and S. Vaidyanathan, Hybrid synchronization of nscroll Chua and Lur'e chaotic systems via backstepping control with novel feedback, Archives of Control Sciences, vol. 22, pp. 343-365 (2012).

125. R. Suresh and V. Sundarapandian, Global chaos synchronization of a family of n-scroll hyperchaotic Chua circuits using backstepping control with recursive feedback, Far East Journal of Mathematical Sciences, vol. 73, pp. 73-95 (2013).

126. S. Rasappan and S. Vaidyanathan, Hybrid synchronization of n-scroll chaotic Chua circuits using adaptive backstepping control design with recursive feedback, Malaysian Journal of Mathematical Sciences, vol. 7, pp. 219-246 (2013).

127. S. Vaidyanathan and S. Rasappan, Global chaos synchronization of n-scroll Chua circuit and Lur'e system using backstepping control design with recursive feedback, Arabian Journal for Science and Engineering, vol. 39, pp. 3351-3364 (2014).

128. S. Rasappan and S. Vaidyanathan, Global chaos synchronization of WINDMI and Coullet chaotic systems using adaptive backstepping control design, Kyungpook Mathematical Journal, vol. 54, pp. 293-320 (2014).

129. H.T. Yau, Chaos synchronization of two uncertain chaotic nonlinear gyros using fuzzy sliding mode control, Mechanical Systems and Signal Processing, vol. 22, pp. 408-418 (2008).

130. H. Li, X. Liao, C. Li, and C. Li, Chaos control and synchronization via a novel chatter free sliding mode control strategy, Neurocomputing, vol. 74, pp. 3212-3222 (2012).

131. S. Vaidyanathan and S. Sampath, Global chaos synchronization of hyperchaotic Lorenz systems by sliding mode control, Communications in Computer and Information Science, vol. 205, pp. 156-164 (2011).

132. V. Sundarapandian and S. Sivaperumal, Sliding controller design of hybrid synchronization of four-wing chaotic systems, International Journal of Soft Computing, vol. 6, pp. 224-231 (2011).

133. S. Vaidyanathan and S. Sampath, Anti-synchronization of fourwing chaotic systems via sliding mode control, International Journal of Automation and Computing, vol. 9, pp. 274-279 (2012).

134. S. Vaidyanathan, Global chaos synchronisation of identical Li$\mathrm{Wu}$ chaotic systems via sliding mode control, International Journal of Modelling, Identification and Control, vol. 22, no. 2, pp. 170-177 (2014).

135. H.K. Khalil, Nonlinear System, $3^{\text {rd }}$ ed.,Prentice Hall,New Jersey, USA (2002). 